\title{
Evaluation of Quality Factors for the Captured Facial Image
}

\author{
Abhay Goyal \\ M.Tech. Student \\ Department of ECE \\ SBSSTC, Ferozepur, Pujnab
}

\author{
Vikram Mutneja \\ Assistant Professor \\ Department of ECE \\ SBSSTC, Ferozepur, Pujnab
}

\begin{abstract}
In today's digital world, the scenario of the images has totally changed. We can do a lot of computation on the captured facial image for improving its quality. This is possible because of the availability of large number of digital platforms. These platforms have made the computational task easier and less time consuming. Instead of improving the quality of the captured image, we can firstly evaluate the quality of the image by making computation for the selected quality factors. After the evaluation, a proper decision can be made for the factors that need more improvement. Here we have selected sharpness, brightness, luminance, contrast and focus as quality factors which we consider more important for quality estimation and then these factors are calculated using the efficient method.
\end{abstract}

\section{General Terms}

Face recognition, Face extraction, Quality factors, Quality Measures.

\section{Keywords}

Quality factors and Facial image.

\section{INTRODUCTION}

Quality estimation in terms of an image means to quantify the various quality factors which are helpful in computing the quality of an image. In present date, quality estimation has become important because of the widespread use of the surveillance cameras [2]. Almost every place is under surveillance and for security reason people are using high quality cameras. Installing high quality cameras is not enough because the image taken by the surveillance camera may or may not be reliable for identification purpose and this can be due to various reasons. Sometimes the availability of the light highly affects the quality of the captured image. The image is also affected by the blurring effect because the person under surveillance can be at rest or in motion. The quality of the captured image is also affected largely by the pose of the image.

Image quality estimation can be basically divided into two parts [1]: a) "Full-reference" and b) "No-reference" or "blind", in case of full-reference method the quality of the captured image is computed in reference with an ideal image and this method is not very affective in real-time applications. On the other hand, blind reference method is the most favourable method because in this method quality of the image is estimated as an independent entity.

The paper is organized as follows: Introduction in Section 1, Literature Review in Section 2, Measuring quality Factors in Section 3, Methodology in Section 4, Conclusion and Future Scope in Section 5.

\section{LITERATURE REVIEW}

A lot of research has been done in the field of quality estimation of the images but still this area is popular. Here the stress has been laid to summarize the concept of different authors who has worked in this field.

K. Nasrollahi and Thomas B. et al. [2], had used four quality factors i.e. pose-estimation, sharpness, brightness and image resolution. Here pose-estimation is calculated by taking the difference between the centre of the mass and the centre of the detected face region. Average value of sharpness for the facial part of the image and its low-pass filtered image gives us the sharpness. Brightness is taken here as the average value of the illumination component of all of the pixels in the facial region of the image. The image resolution is calculated with respect to the width and height of the face.

M. Ann, A. Ross, A. Abaza and T. Bourlai et al. [4], had worked on illumination, focus and sharpness, contrast and brightness. Contrast is taken to be the difference in colour intensities of the image and is calculated as RMS parameter. Brightness is calculated as the average of the brightness components by firstly converting the image in HSB domain. Focus here is calculated by taking the average of the $L_{1}$-norm of the image and the energy of the Laplacian of the image. Image sharpness is calculated by the gradient method and illumination is calculated as the weighted sum of the mean intensity values of the image divided into $\left(4^{*} 4\right)$ blocks.

D. Bhattacharjee, S. Prakash and P. Gupta et al. [7], had used Eye Detection, Sharpness, Noise, Luminance and Contrast for working on quality assessment. For eye detection image clustering method is used. Sharpness is computed using Just Noticeable Blur (JNB) which provides a localized threshold for the perceived blurriness at a given contrast level. By taking the absolute vertical and horizontal difference of the pixels in the non edge parts of the image, noise is calculated. Contrast can be computed with the RMS method which is basically the standard deviation of the pixel intensities. Here luminance is calculated by changing RGB colorspace to $\mathrm{YC}_{\mathrm{b}} \mathrm{C}_{\mathrm{r}}$ colorspace, here $\mathrm{Y}$ component when normalized over the intensity range of the image gives the luminance.

Teruaki, Yuki and Osamu et al., [5], had proposed a humanface extraction technique using the HSV colour model and had confirmed the effectiveness of this method experimentally. The proposed method consists of six steps: input of images, extraction of moving areas, extraction of skin colour areas, extraction of facial area, extraction of facial parts and setting of the specific area on a face.

Nguyen, Van Huan and Hakil et al., [8], had proposed a method in which they had used Background segmentation, Skin detection, Eye detection, Lip feature detection and Chin detection as parameters. In this paper, a facial image preprocessing method is proposed that utilizes both edge and 
color information. The use of edge-based segmentation method makes the background segmentation algorithm independent to background color.

\section{MEASURING QUALITY FACTORS}

All the methods that are used today are using the similar way to compute the quality factors. Out of various quality factors available for a given image, we consider the evaluation of contrast, brightness, sharpness, illumination and focus $[7,4]$ as most commonly used because of their importance in evaluation of the overall quality of the captured facial image $[4,1]$.

\subsection{Quality factors}

Quality factors give us the correct information about the overall quality of the input image. After deciding the quality factors, the next step is to evaluate them using the appropriate method out of the various available methods. For the evaluation of selected quality factors, we have used the following approach:

\subsubsection{Sharpness}

Sharpness is one of the most important photographic image quality factor because it gives the amount of detail an imaging system can reproduce [4]. Sharpness gives the clarity of detail in a facial image, or it gives the degree of clarity of an image. Here we have measured the Sharpness $(S)$ using the gradient technique [23].

$$
S=\sum_{x=1}^{M-2}\left[\sum_{y=1}^{N-2} G(x, y)\right]
$$

\subsubsection{Brightness}

The brightness $(B)$ of the facial image can be calculated by converting it into the HSB (hue, saturation and brightness) domain [16]. Firstly the RGB image is normalized to [0, 1] range and then it is converted to HSB domain using the below given method.

$$
\begin{gathered}
{\left[\begin{array}{rrr}
r & g & b
\end{array}=1 / 255 *[\mathrm{R} \mathrm{G} \mathrm{B}]\right.} \\
B=(1 / M N) \sum_{x=1}^{M}\left[\sum_{y=1}^{N}[\max (r, g, b)]\right]
\end{gathered}
$$

\subsubsection{Luminance}

Luminance is defined as the intensity of light emitted from a unit area surface in a given direction. In terms of image, it gives the amount of light that is emitted from a particular area [4]. Luminance for an RGB image is calculated as a weighted sum of the red $(R)$, blue $(B)$ and green $(G)$ components [7]. The image is converted into $\mathrm{YC}_{\mathrm{b}} \mathrm{C}_{\mathrm{r}}$ colorspace from RGB colorspace as:

$$
\begin{gathered}
Y=16+(65.4 * R+128.5 * G+25 * B) \\
C_{b}=128+(-37.7 * R-74.2 * G+112 * B) \\
C_{r}=128+(112 * R-93.7 * G-18.2 * B)
\end{gathered}
$$

Luminance is calculated as mean of the $Y$ component normalized over the intensity range of the facial image.

\subsubsection{Contrast}

Contrast is the difference in luminance or colour intensities that makes an object (a facial image) distinguishable. Simply we can say that contrast is the difference between the maximum and minimum pixel intensity of an image. Contrast of a given image is calculated using the following equation [23]:

$$
C_{R M S}=\frac{\sqrt{\sum_{x=1}^{M}\left[\sum_{y=1}^{N}\{I(x, y)-\mu\}^{2}\right]}}{M N}
$$

where $\mu$ represents the mean intensity value of the input facial image $I(x, y)$ of size $N * M$.

\subsubsection{Focus}

Image focus refers to the degree of blur present in the image. Here image focus is calculated using the average of the $L_{1}$ norm and the energy of the Laplacian of the image [18]. The $L_{1}$ - norm of the image is given as:

$$
F_{L_{1}}=\sum_{x=1}^{M}\left[\sum_{y=1}^{N}\left|G_{x x}(x, y)\right|+\left|G_{y y}(x, y)\right|\right]
$$

and the energy of the Laplacian of the image is given as:

$$
F_{E L}=\sum_{x=1}^{M}\left[\sum_{y=1}^{N}\left|G_{x x}(x, y)\right|+\left|G_{y y}(x, y)\right|\right]^{2}
$$

where $G_{x x}$ and $G_{y y}$ are the second derivatives in the horizontal and vertical directions. Here focus is taken as the average of $F_{L_{1}}$ and $F_{E L}$.

\section{METHODOLOGY}

We have selected a group of images and used MATLAB$2013 \mathrm{~b}$ platform to carry out all our programming (see Figure 2). A brief description of the working of above model is given below:

a) The first step is to run Matlab-2013b on your machine. The command window will appear. Type "guide" keyword in this window. This will open GUIDE Quick Start dialog box. Click on the Create New GUI tab and select blank GUI template. Then a blank GUI will open. Basically a GUI is a graphical display in one or more windows containing controls, called components. This enables a user to perform interactive tasks.

b) In the GUI we have used various controls that make our GUI simple in operation. Here we have used push buttons, axes and static text fields for our GUI.

c) The working of this GUI starts by clicking the "LOAD IMAGE" button. Then a Select image dialogue box opens. From this select the appropriate database that contains the test images. After selecting an image from the database, the selected image will be displayed on the first axes. 


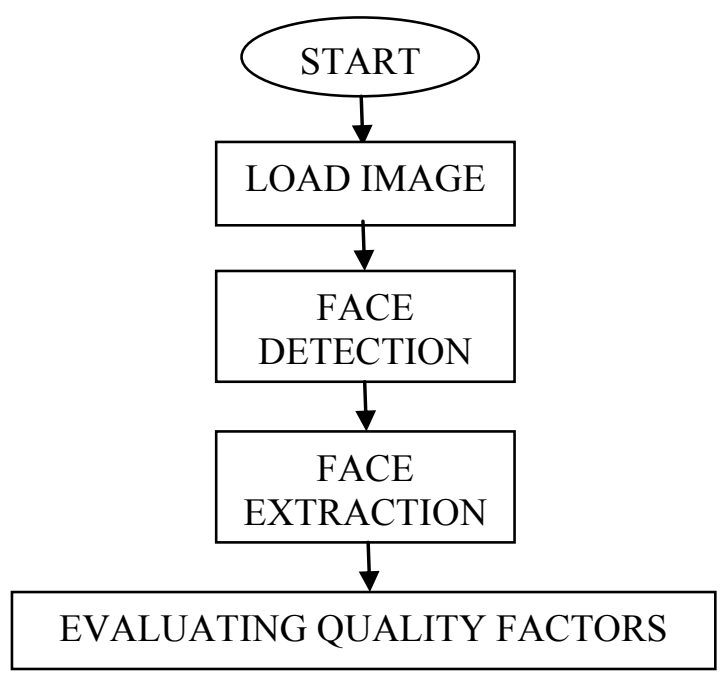

Fig 1: Block Diagram of the proposed work

d) Now the next step is to detect the face in the selected image. For this click on "DETECT FACE" button. This is done here by using vision.CascadeObjectDetector to detect the location of a face in an image. The cascade object detector uses the Viola-Jones algorithm and a trained classification model for detection. After detection of the facial part then comes the step to display it. This is done by using bounding box which gives us a red colored box around the detected facial region. The face detected image is displayed using a separate axes control.

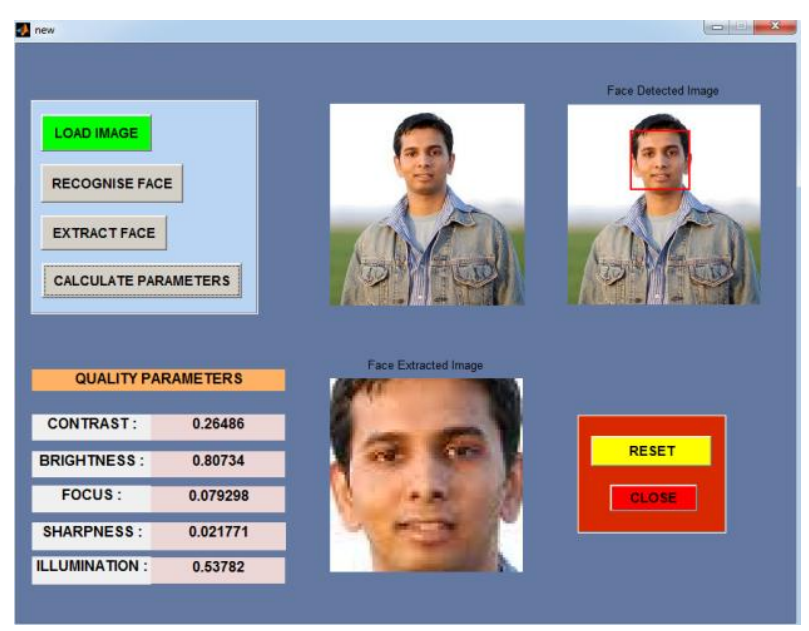

Fig 2: The Proposed GUI

e) Now when the face is detected then we have to extract the facial part for carrying out computation for the selected quality parameters. This is done by clicking on "EXTRACT FACE" button. The code behind this push button is basically using the command imcrop $(I,[B B(i,:)])$, here $I$ represents the face detected image and $B B$ (Bounding Box) represents the bounding box. Also this cropped facial image is shown using a separate axes control.

f) After all the above mentioned steps are completed, then the last step is to calculate the selected quality parameters. This is done by clicking on "CALCULATE PARAMETERS" button. Behind this button, the programming is done according to the selected methods given in section III of this paper. After the calculation of these factors, these are displayed on the GUI using static text fields.

\section{CONCLUSION AND FUTURE SCOPE}

By making computation for the selected quality factors from the techniques mentioned in this paper we can make a very good estimate about the various quality parameters. In future we are preparing to work on combining these quality factors and to propose a quality index which will help in distinguishing the set of good images from the bad set of images.

\section{ACKNOWLEDGMENTS}

Thanks to my Guide and my family members who always support, help and guide me during my thesis. Special thanks to my mother who always support my innovative ideas.

\section{REFERENCES}

[1] Ayman Abaza, Mary Ann Harrison, Thirimachos Bourlai, "Quality Metrics for Practical Face Recognition", in 21st International Conference on Pattern Recognition (ICPR 2012), pp. 3103-3107,11-15 Nov. 2012.

[2] Kamal Nasrollahi, Thomas B. Moeslund, "Face Quality Assessment System in Video Sequences", Biometrics and Identity Management Lecture Notes in Computer Science, vol. 5372, pp. 10-18, 2008.

[3] Jiansheng Chen, Member, IEEE, Yu Deng, Gaocheng Bai and Guangda Su, "Face Image Quality Assessment Based on Learning to Rank", in IEEE Signal Processing Letters, vol. 22,No. 1, 2015, pp. 90-94.

[4] Ayman Abaza, Mary Ann Harrison, Thirimachos Bourlai, Arun Ross, "Design and evaluation of photometric image quality measures for effective face recognition", Biometrics, IET, vol. 3, Issue: 4, pp. 314324

[5] Teruaki Hirano, Yuki Nakagawa and Osamu Nakamura, "Highly Accurate Extraction of faces and facial parts taking into consideration people with glasses and the specific areas of the face for extracting specific features used in the recognition of facial expressions", Electrical and Computer Engineering, 23rd Canadian Conference, pp. 1-7, 2010.

[6] Mohammad A. Haque, Kamal Nasrollahi and Thomas B. Moeslund, "Constructing Facial Expression Log from Video Sequences using Face Quality Assessment", in Proceedings of the 9th International Conference on Computer Vision Theory and Applications, 2014, pp. 517-525.

[7] Debalina Bhattacharjee, Surya Prakash, and Phalguni Gupta, "No-Reference Image Quality Assessment for Facial Images", in Advanced Intelligent Computing Theories and Applications. With Aspects of Artificial Intelligence Lecture Notes in Computer Science, vol. 6839, pp. 594-601, 2012.

[8] Nguyen Thi Hai Binh, Nguyen Van Huan and Hakil Kim, "Combination of Edge and Color Information for Robust Preprocessing in Facial Image Quality Assessment", in Systems Man and Cybernetics (SMC) IEEE International Conference, pp. 3594-3600, 2010.

[9] Rein-Lien Vincent Hsu, Jidnya Shah, Brian Martin, "Quality Assessment of Facial Images", Biometric Consortium Conference, Biometrics Symposium: Special Session on Research, pp. 1-6, 2006. 
[10] Krzysztof Kryszcsuk and Andrzej Drygajlo, "On Combining Evidence for Reliability Estimation in Face Verification", in 14th European Signal Processing Conference, pp. 1-5, 2006.

[11] P. Jonathon Phillips, J. Ross Beveridge, Bruse A. Draper, Alive J. O'Toole, Geof Givens ,et al, "An Introduction to the Good, the Bad, \& the Ugly Face Recognition Challenge Problem", in IEEE International Conference on Automatic Face \& Gesture Recognition and Workshops (FG 2011), pp. 346-353, 2011.

[12] Jianfeng Long and Shutao Li, "Near Infrared Face Image Quality Assessment System of Video Sequences", in Sixty International Conference on Image and Graphics, pp. 275-279, 2011

[13] Z. Wang and A. Bovik, "A universal image quality index", IEEE Signal Processing Letters, 9:81-84, 2002.

[14] T. Ahonen, A. Hadid, and M. Pietikainen, "Face description and local binary patterns: Application to face recognition", IEEE Trans. Pattern Anal. Mach. Intell., vol. 28, no. 12, pp. 2037-2041, Dec. 2006.

[15] Yuridia, O., Castillo, G., "Survey About Facial Image Quality", Fraunhofer Institute of Computer Graphics Research, 2006.

[16] Bezryadin, S., Bourov, P., Ilinih, D., "Brightness calculation in digital image processing", in International Symp. on Technologies for Digital Fulfillment, 2007.
[17] K. Kryszczuk and A. Drygajlo., "Impact of combining quality measures on biometric sample matching", in proc. of the IEEE BTAS, 2009.

[18] Yap, P.-T., Raveendran, P., "Image focus measure based on Chebyshev moments", in IEE Proc. Vis. Image Signal Process., pp. 128-136, 2004.

[19] A. Fourney and R. Laganiere, "Constructing Face Image Logs that are Both Complete and Concise", in 4th Canadian Conference on Computer Vision and Robot Vision, pp. 488-494, 2007.

[20] F. Weber, "Some quality measures for face images and their relationship to recognition performance", in Biometric Quality Workshop. National Institute of Standards and Technology, 2006.

[21] Briechle, K., and Hanebeck, "Template Matching using Fast Normalized Cross Correlation", in Proc. of SPIE Aero Sense Symposium, vol. 43-87, pp. 1-8, 2001.

[22] Marques, O., "Practical Image and Video Processing Using MATLAB", in Wiley-IEEE Press, 2011.

[23] Gao, X., Ki, S.Z., Liu, R., Zhang, "Standardization of face image sample quality", in Int. Conf. on Biometrics (ICB), 2007.

[24] Gao, X., Ki, S.Z., Liu, R., Zhang, "The CAS-PEAL large-scale Chinese face database and baseline evaluations", in IEEE Trans. Syst. Man Cybern., 38, pp. 149-161, 2008 